\title{
Perfect set properties in models of ZF
}

\author{
by
}

\section{A. Di Prisco and F. C. Galindo (Caracas)}

\begin{abstract}
We study several perfect set properties of the Baire space which follow from the Ramsey property $\omega \rightarrow(\omega)^{\omega}$. In particular we present some independence results which complete the picture of how these perfect set properties relate to each other.
\end{abstract}

1. Introduction. We will study some partition properties of the spaces $\omega^{\omega}$ and $[\omega]^{\omega}$ defined in terms of different kinds of perfect sets. To state these properties, we give some definitions. The set of finite sequences of natural numbers is denoted by $\omega^{<\omega}$, and $\omega^{\omega}$ denotes the set of all infinite sequences of natural numbers. The Baire space is the set $\omega^{\omega}$, with the product topology, and $2^{\omega}:=\{f: f: \omega \rightarrow 2\}$ with the product topology is the Cantor space. Let $[\omega]^{\omega}$ be the family of all infinite subsets of $\omega$; this set with the topology inherited from $2^{\omega}$ is homeomorphic to the Baire space. For any $A \in[\omega]^{\omega}$ we use $[A]^{\omega}$ to denote the set of all infinite subsets of $A$. We now list the partition properties we will be dealing with.

The Ramsey property: A set $\mathcal{A} \subseteq[\omega]^{\omega}$ is Ramsey if there is $A \in[\omega]^{\omega}$ such that $[A]^{\omega} \subseteq \mathcal{A}$ or $[A]^{\omega} \cap \mathcal{A}=\emptyset$. The Ramsey property, expressed by the symbol $\omega \rightarrow(\omega)^{\omega}$, is the statement that every $\mathcal{A} \subseteq[\omega]^{\omega}$ is Ramsey.

The sublattice property: Let $K, H \in[\omega]^{\omega}$ be such that $K \subseteq H$ and $H \backslash K$ is also infinite. We put $[K, H]=\{Y \subseteq \omega: K \subseteq Y \subseteq H\}$. Clearly, $([K, H], \subseteq)$, is a sublattice of $\mathcal{P}(\omega)$. The sublattice property [8] denoted by $\omega \rightarrow((\omega))^{\omega}$ is the following: For every $\mathcal{A} \subseteq[\omega]^{\omega}$ there is a sublattice $[K, H]$ such that $[K, H] \subseteq \mathcal{A}$ or $[K, H] \cap \mathcal{A}=\emptyset$. This property has been called the doughnut property in previous articles. The sublattices of the form $[K, H]$ as above are related to Silver forcing, for this reason a set $\mathcal{A} \subseteq[\omega]^{\omega}$ for which given a sublattice $\left[K^{\prime}, H^{\prime}\right]$ there is a sublattice $[K, H] \subseteq\left[K^{\prime}, H^{\prime}\right]$ such that $[K, H] \subseteq \mathcal{A}$ or $[K, H] \cap \mathcal{A}=\emptyset$ is said to be Silver measurable (see [3, 10]).

2010 Mathematics Subject Classification: 03E02, 03E25, 03E35.

Key words and phrases: perfect set property, Ramsey property, models of ZF. 
The polarized partition property: The notation

$$
\left(\begin{array}{c}
\omega \\
\omega \\
\vdots
\end{array}\right) \rightarrow\left(\begin{array}{c}
n_{0} \\
n_{1} \\
\vdots
\end{array}\right)
$$

means that for every $F: \omega^{\omega} \rightarrow 2$ there is a sequence $\left\{H_{i}\right\}_{i \in \omega}$ of finite sets such that

- $H_{i} \subseteq \omega,\left|H_{i}\right|=n_{i}$,

- $F$ is constant on $\prod_{i \in \omega} H_{i}$.

The Bernstein property: Recall that a non-empty subset of $\omega^{\omega}$ is perfect if it is closed and has no isolated points. The Bernstein property is the statement: For every $\mathcal{A} \subseteq \omega^{\omega}$ there is a perfect set $P \subseteq \omega^{\omega}$ contained in $\mathcal{A}$ or disjoint from $\mathcal{A}$. This property has been denoted $\omega \rightarrow$ (perfect) $^{\omega}$ (see [5]).

It is easy to verify that the Ramsey property implies both the sublattice property and the polarized property, and that each of these two implies the Bernstein property. The Axiom of Choice provides a counterexample to the Bernstein property ([1]), and thus to all of the properties mentioned, but they all hold in Solovay's model ([14]). Solovay's model is constructed starting from a model with an inaccessible cardinal, and it is not known if a model for the Ramsey property can be found without assuming the existence of such a cardinal. Nevertheless, the consistency of the sublattice property with ZF + DC does not require this inaccessibility hypothesis (it holds, for example, in Shelah's model where every set of reals has the Baire property [15]).

In this article we present two main results:

(1) There is a model of ZF, which we call $N_{1}$, satisfying the sublattice property where the polarized partition property does not hold. This model is obtained, following Truss [17] and Feferman [9], by constructing $L(A)$ in a forcing extension of the constructible universe $L$ with an infinite collection $A$ of Cohen generic reals. This answers a question of [7].

It is pertinent to mention that the notation $L(X)$ has been used in the literature to denote various ways to relativize the concept of constructibility. Here, for any class $X$, we use $L(X)$ to denote a relativized model which is defined by setting $L_{0}(X)=\emptyset, L_{\lambda}(X)=\bigcup_{\alpha<\lambda} L_{\alpha}(X)$ for limit ordinals $\lambda$, and $L_{\alpha+1}(X)=$ the collection of all subsets of $L_{\alpha}(X)$ definable in $L_{\alpha}(X)$ using $\in$, parameters from $L_{\alpha}(X)$, and unary predicates for $x \cap L_{\alpha}(X)$ for all $x \in X$ (see [2, 9]).

A model given in 8 ] shows that the polarized partition property does not imply the sublattice property. Thus, neither of these properties implies the other. It follows that the Bernstein property does not imply the polarized partition property, which answers a question posed in [6, 5]. 
(2) There is a model of ZF, which we call $N_{2}$, where there is an $\aleph_{1^{-}}$ sequence of reals, and the sublattice property holds. In this model there is a non-meager filter on $\omega$, which implies that the Ramsey property fails.

We conclude that $\aleph_{1} \leq 2^{\omega}$ does not imply the existence of a Bernstein set (where $\aleph_{1} \leq 2^{\omega}$ means that there is an injection of $\aleph_{1}$ into the set of real numbers). Recall that a set of reals is said to have the perfect set property if it is either countable or contains a perfect subset. It is well known that $\aleph_{1} \leq 2^{\omega}$ implies the existence of an uncountable set of real numbers without a perfect subset, thus $\aleph_{1} \leq 2^{\omega}$ is a weak choice principle discriminating between the perfect set property and the Bernstein property.

The paper is organized as follows. In Section 2 we describe the relativization $L(A)$ with the properties mentioned above. Section 3 is devoted to defining the model $N_{1}$ and the proof that it has the sublattice property but not the polarized property. In Section 4 we define the model $N_{2}$ and prove that in this model the sublattice property holds, there is a non-meager filter and, as a consequence, the Ramsey property does not hold.

These results together with [8] show that the only implications that hold between these properties are the ones given in the following diagram.

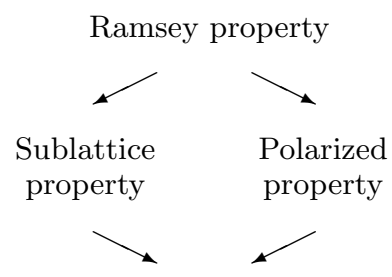

Bernstein property

\section{Relativized constructibility. The models $L(A)$}

2.1. Definition of the models of the form $L(A)$. Given a set $A$, the class $L(A)$ is defined by transfinite induction as follows:

DEFINITION 1.

$$
\begin{aligned}
& L_{0}(A):=\emptyset, \\
& L_{\alpha+1}(A):=\left\{X \subseteq L_{\alpha}(A): X\right. \text { is definable in the structure } \\
&\left.\left(L_{\alpha}(A), \in,\left\langle a \cap L_{\alpha}(A): a \in A\right\rangle,\left\langle d: d \in L_{\alpha}(A)\right\rangle\right)\right\}, \\
& L_{\lambda}(A):=\bigcup_{\beta \in \lambda} L_{\beta}(A), \quad \lambda \text { limit, } \\
& L(A):=\bigcup_{\alpha \in \text { Ord }} L_{\alpha}(A) .
\end{aligned}
$$

For the successor step, the expression " $X$ is definable in the structure $\left(L_{\alpha}(A), \in,\left\langle a \cap L_{\alpha}(A): a \in A\right\rangle,\left\langle d: d \in L_{\alpha}(A)\right\rangle\right)$ " implicitly requires that 
we have a first order language with identity with constants for the elements of $L_{\alpha}(A)$, a unary relation symbol $P_{a}$ for each set $a \cap L_{\alpha}(A), a \in A$, and a binary relation symbol for $\in$. As usual, this definition of $L(A)$ can be formalized in $\mathrm{ZF}$, for example, by modifying appropriately the construction of $L$ in [11] as follows.

Definition 2. Let $F$ be a set, $n \in \omega(n>0)$ and $i, j<n$.

(a) $\operatorname{Diag}_{\in}(F, n, i, j):=\left\{s \in F^{n}: s(i) \in s(j)\right\}$.

(b) $\operatorname{Diag}_{=}\left\{(F, n, i, j):=\left\{s \in F^{n}: s(i)=s(j)\right\}\right.$.

(c) $\operatorname{Proj}(F, R, n):=\left\{s \in F^{n}: \exists t \in R(t\lceil n=s)\}\right.$.

(d) $\operatorname{Pred}(F, n, i, a):=\left\{s \in F^{n}: s(i) \in a \cap F\right\}$ for every $a \in A$.

(e) By recursion on $k \in \omega$, define $\operatorname{Df}_{A}^{\prime}(k, F, n)$, simultaneously for all $n$ :

(1) $\operatorname{Df}_{A}^{\prime}(0, F, n):=\left\{\operatorname{Diag}_{\in}(F, n, i, j): i, j<n\right\} \cup\left\{\operatorname{Diag}_{=}(F, n, i, j):\right.$ $i, j<n\} \cup\{\operatorname{Pred}(F, n, i, x): x \in A\}$.

(2) $\mathrm{Df}_{A}^{\prime}(k+1, F, n):=\mathrm{Df}_{A}^{\prime}(k, F, n) \cup\left\{F^{n}-R: R \in \mathrm{Df}_{A}^{\prime}(k, F, n)\right\}$ $\cup\left\{R \cap S: R, S \in \operatorname{Df}_{A}^{\prime}(k, F, n)\right\} \cup\left\{\operatorname{Proj}(F, R, n): R \in \operatorname{Df}_{A}^{\prime}(k, F\right.$, $n+1)\}$.

(f) $\operatorname{Df}_{A}(F, n):=\bigcup_{k \in \omega} \operatorname{Df}_{A}^{\prime}(k, F, n)$.

Now, we define the operation $\mathcal{D}_{A}(F)$.

Let $X$ be a set, and $s=\left\langle s_{0}, \ldots, s_{m-1}\right\rangle$ and $t=\left\langle t_{0}, \ldots, t_{n-1}\right\rangle$ two finite sequences of elements of $X$. Denote by $s \frown t$ the concatenation of $s$ and $t$, that is,

$$
s \frown t:=\left\langle s_{0}, \ldots, s_{m-1}, t_{0}, \ldots, t_{n-1}\right\rangle .
$$

Definition 3. Given a set $F$ define

$$
\begin{aligned}
\mathcal{D}_{A}(F):=\left\{X \subseteq F: \exists n \in \omega \exists s \in F^{n} \exists R \in \operatorname{Df}_{A}(F, n+1)\right. \\
(X=\{z \in F: s \frown(z) \in R\})\} .
\end{aligned}
$$

Finally,

Definition 4. $L(A)$ is defined by transfinite induction as follows:

$$
\begin{aligned}
L_{0}(A) & :=\emptyset, \\
L_{\alpha+1}(A) & :=\mathcal{D}_{A}\left(L_{\alpha}(A)\right), \\
L_{\lambda}(A) & :=\bigcup_{\beta \in \lambda} L_{\beta}(A), \quad \lambda \text { limit, } \\
L(A) & :=\bigcup_{\alpha \in \text { Ord }} L_{\alpha}(A) .
\end{aligned}
$$

For $x \in L(A)$, the rank of $x$ in $L(A), \operatorname{rank}_{L(A)}(x)$, is the least ordinal $\alpha$ such that $x \in L_{\alpha+1}(A)$. The rank of $x$ in the universe $V$ is denoted by $\operatorname{rank}(x)$. 
Proposition 5. $L(A)$ is a transitive model of ZF containing the ordinals.

Proof. As for $L$ (see, for example, [11]).

Proposition 6. If $A$ is transitive, then $A \subseteq L(A)$.

Proof. By induction on the rank of $A$ in the universe $V$.

Thus, when $A$ is transitive, $L(A)$ is the smallest transitive model containing $A$ and the ordinals.

2.2. The model $L(A)$ when $A$ is an infinite set of Cohen reals. Let $M$ be a transitive model of ZFC, and let $M[G]$ be the generic extension obtained by using Cohen forcing to add $\kappa \geq \omega$ generic reals, namely, the poset

$$
\mathbb{C}_{\kappa}:=\{p:|p|<\omega \wedge p \subseteq \kappa \times \omega \times 2\}, \quad p \leq q \leftrightarrow q \subseteq p .
$$

Let $\beta \in \kappa$. Define

$$
a_{\beta}:=\{m \in \omega: \exists p \in G(p(\beta, m)=1)\} .
$$

Each generic real $a_{\beta}$ has a canonical name $\bar{a}_{\beta}=\{(\check{n}, p): p(\beta, n)=1\}$ and the set $A=\left\{a_{\beta}: \beta<\kappa\right\}$ also has the canonical name $\left\{\left(\bar{a}_{\beta}, 1\right): \beta<\kappa\right\}$

We now consider $L(A)$ computed in $M[G]=M\left[\left\{a_{\beta}: \beta<\kappa\right\}\right]$. We prove that $A \notin L(A)$, although each $a_{\beta}$ is, of course, in $L(A)$, and that the Axiom of Choice does not hold in $L(A)$.

Proposition 7. $A \notin L(A)$.

Proof. Suppose towards a contradiction that $A \in L(A)$, and let $\operatorname{rank}_{L(A)}(A)=\alpha$. Then

$$
\begin{aligned}
A=\left\{z \in L_{\alpha}(A)\right. & : \\
M[G] & \left.\models \varphi^{L_{\alpha}(A)}\left(z, b_{0}, \ldots, b_{n-1}, a_{\xi_{0}} \cap L_{\alpha}(A), \ldots, a_{\xi_{m-1}} \cap L_{\alpha}(A)\right)\right\},
\end{aligned}
$$

where $b_{i} \in L_{\alpha}(A)$ for every $i<n$, and $a_{\xi_{j}} \in A$ for every $j<m$.

Since $a_{\xi_{j}} \cap L_{\alpha}(A)=a_{\xi_{j}}$ for every $j \in m$, each $b_{i}, i \in n$, can be replaced by its definition, and also $L_{\alpha}(A)$ can be replaced by its definition. Thus, the formula $\varphi^{L_{\alpha}(A)}$ can be rewritten to define $A$ by a formula $\Phi$ of the form

$$
A=\left\{z \in L_{\alpha}(A): M[G] \models \Phi\left(z, a_{\delta_{0}}, \ldots, a_{\delta_{k-1}}\right)\right\},
$$

where each $a_{\delta_{j}}(j \in k)$ is a generic real.

Let $a_{\beta}$ be a generic real different to $a_{\delta_{0}}, \ldots, a_{\delta_{k-1}}$. There is a condition $p \in G$ such that $p \Vdash \Phi\left(\bar{a}_{\beta}, \bar{a}_{\delta_{0}}, \ldots, \bar{a}_{\delta_{k-1}}\right)$. We define an automorphism $\pi_{X}: \mathbb{C}_{\kappa} \rightarrow \mathbb{C}_{\kappa}$ as follows: let $X=\alpha \times \omega \backslash\left(\left(\left\{\delta_{0}, \ldots, \delta_{k-1}\right\} \times \omega\right) \cup \operatorname{dom}(p)\right)$, and put

$$
\pi_{X}(q)(\gamma, n):= \begin{cases}q(\gamma, n) & \text { if }(\gamma, n) \notin X \\ 1-q(\gamma, n) & \text { if }(\gamma, n) \in X\end{cases}
$$


Notice that $p=\pi_{X}(p)$. By symmetry,

$$
\pi_{X}(p) \Vdash \Phi\left(\pi\left(\bar{a}_{\beta}\right), \bar{a}_{\delta_{0}}, \ldots, \bar{a}_{\delta_{k-1}}\right),
$$

since $\pi\left(\bar{a}_{\delta_{i}}\right)=\bar{a}_{\delta_{i}}$ for every $i \in k$. This contradicts that $\operatorname{Val}\left(\pi_{X}\left(\bar{a}_{\beta}\right), G\right)$ is not an element of $A$. In fact, $\operatorname{Val}\left(\pi_{X}\left(\bar{a}_{\beta}\right), G\right) \neq a_{\beta}$ since the intersection $a_{\beta} \cap \operatorname{Val}\left(\pi_{X}\left(\bar{a}_{\beta}\right), G\right)$ is finite. Moreover, $\operatorname{Val}\left(\pi_{X}\left(\bar{a}_{\beta}\right), G\right)$ is different from each of the other generic reals, since for every $\gamma \neq \beta, a_{\gamma} \cap a_{\beta}$ is infinite.

Proposition 8. The Axiom of Choice does not hold in $L(A)$.

Proof. We show by contradiction that in $L(A)$ the set of real numbers cannot be well ordered.

Suppose, to reach a contradiction, that there is a well ordering $\leq$ of the set of real numbers in $L(A)$, and let $\operatorname{rank}_{L(A)}(\leq)=\alpha$. Then there is a formula $\varphi$ such that $\leq$ is the set of all $(x, y) \in L_{\alpha}(A)$ such that

$$
M[G] \models \varphi^{L_{\alpha}(A)}\left(x, y, b_{0}, \ldots, b_{n-1}, a_{\xi_{0}} \cap L_{\alpha}(A), \ldots, a_{\xi_{m-1}} \cap L_{\alpha}(A)\right),
$$

where $b_{i} \in L_{\alpha}(A)$ for every $i<n$, and $a_{\xi_{j}} \in A$ for $j<m$.

As in the previous proof, the formula $\varphi^{L_{\alpha}(A)}$ can be rewritten to obtain a formula of the form

$$
\Phi\left(v, z, a_{\delta_{0}}, \ldots, a_{\delta_{k-1}}\right)
$$

which defines the well ordering $\leq$ in $M[G]$.

Consider the equivalence relation on $2^{\omega} \cap L(A)$ defined by $x \sim y$ if and only if $\exists n \forall m \geq n(x(m)=y(m))$.

We will obtain a contradiction using an automorphism of the forcing partial order which leaves $\leq$ fixed and modifies a certain generic real maintaining its equivalence class.

Let $a_{\eta_{0}}$ a generic real different from the parameters in the formula $\Phi$, and let $\left[a_{\eta_{0}}\right]$ be its equivalence class in $L(A)$. Let $x_{0}$ be the $\leq$-least element of $\left[a_{\eta_{0}}\right]$. Then

$$
\forall y\left[y \subseteq \omega \wedge \exists n \forall m \geq n\left(y(m)=a_{\eta_{0}}(m)\right) \rightarrow x_{0} \leq y\right] .
$$

Let $k_{0} \in \omega$ be such that $\forall k \geq k_{0}\left(k \in x_{0} \leftrightarrow k \in a_{\eta_{0}}\right)$.

There is $p \in G$ such that

$$
\begin{aligned}
(\bullet) \quad p \Vdash \forall y[y \subseteq \check{\omega} \wedge \exists n \forall m \geq n(y(m)= & \left.\left.\bar{a}_{\eta_{0}}(m)\right) \rightarrow \dot{x_{0}} \leq y\right] \wedge \\
& \forall k \geq \check{k_{0}}\left(k \in \dot{x_{0}} \leftrightarrow k \in \bar{a}_{\eta_{0}}\right) .
\end{aligned}
$$

Here $\dot{x_{0}}$ is a $\mathbb{C}_{\kappa}$-name for $x_{0}$.

Let $l \geq k_{0}(l \in \omega)$ be such that $\left(\left(\eta_{0}, l\right), 1\right) \notin p$ and $\left(\left(\eta_{0}, l\right), 0\right) \notin p$, and let $\pi: P \rightarrow \bar{P}$ be the automorphism induced by the function

$$
\pi^{\prime}: \alpha \times \omega \times 2 \rightarrow \alpha \times \omega \times 2
$$

defined by $\pi^{\prime}\left(\left(\eta_{0}, l\right), 1\right)=\left(\left(\eta_{0}, l\right), 0\right), \pi^{\prime}\left(\left(\eta_{0}, l\right), 0\right)=\left(\left(\eta_{0}, l\right), 1\right)$ and the identity elsewhere. Notice that $p=\pi(p)$. 
By symmetry,

$$
\begin{aligned}
\pi(p) \Vdash \forall y[y \subseteq \check{\omega} \wedge \exists n \forall m \geq n(y(m) & \left.\left.=\pi\left(\bar{a}_{\eta_{0}}\right)(m)\right) \rightarrow \pi\left(\dot{x_{0}}\right) \leq y\right] \wedge \\
\forall k & \geq \check{k_{0}}\left(k \in \pi\left(\dot{x_{0}}\right) \leftrightarrow k \in \pi\left(\bar{a}_{\eta_{0}}\right)\right) .
\end{aligned}
$$

But this implies a contradiction, since

(1) $\pi\left(\bar{a}_{\beta}\right)=\bar{a}_{\beta}$ for $\beta \neq \eta_{0}$. In particular $\pi$ fixes the parameters of the formula that defines the well ordering $\leq$.

(2) $l \in a_{\eta_{0}}=\operatorname{Val}\left(\bar{a}_{\eta_{0}}, G\right) \leftrightarrow l \notin \operatorname{Val}\left(\pi\left(\bar{a}_{\eta_{0}}\right), G\right)$.

(3) If $m \neq l$, then $m \in a_{\eta_{0}} \leftrightarrow m \in \operatorname{Val}\left(\pi\left(\bar{a}_{\eta_{0}}\right), G\right)$.

Note that (2) and (3) imply that $a_{\eta_{0}}=\operatorname{Val}\left(\bar{a}_{\eta_{0}}, G\right)$ and $\operatorname{Val}\left(\pi\left(\bar{a}_{\eta_{0}}\right), G\right)$ differ only on $l$, thus

(4) $\left[a_{\eta_{0}}\right]=\left[\operatorname{Val}\left(\pi\left(\bar{a}_{\eta_{0}}\right), G\right)\right]$.

(5) $x_{0}=\operatorname{Val}\left(\pi\left(\dot{x_{0}}\right), G\right)$, by the left parts of the propositions forced in $(\bullet)$ and $(\circ)$ and by (4).

(6) $x_{0} \neq \operatorname{Val}\left(\pi\left(\dot{x_{0}}\right), G\right)$, by (2) and (3) together with the right parts of the propositions forced in $(\bullet)$ and $(\circ)$.

Obviously, (5) and (6) contradict each other.

3. Independence of the sublattice property from the polarized property. Let $L\left[\left\{a_{\xi}: \xi<\omega_{1}\right\}\right]$ be a generic extension of $L$ obtained by adding $\aleph_{1}$-many Cohen generic reals to $L$, and let $N_{1}=L\left(\left\{a_{\xi}: \xi<\omega_{1}\right\}\right)$ computed in the generic extension.

We will show that in $N_{1}$ the property $\omega \rightarrow((\omega))^{\omega}$ holds, but not the polarized partition relation

$$
\left(\begin{array}{c}
\omega \\
\omega \\
\vdots
\end{array}\right) \rightarrow\left(\begin{array}{c}
2 \\
2 \\
\vdots
\end{array}\right) .
$$

This answers a question of [7].

In 8 it is established that the above displayed relation does not imply $\omega \rightarrow((\omega))^{\omega}$. So, neither of these properties implies the other.

For $s, t \in 2^{<\omega}$, we write $s \sqsubseteq t$ when $s$ is an initial segment of $t$, and $s \sqsubset t$ when $s$ is a proper initial segment of $t$. As usual, $T \subseteq 2^{<\omega}$ is a tree if $t \in T$ and $s \sqsubset t$ implies $s \in T$. Given a tree $T \subseteq 2^{<\omega}$, we say $T$ is uniform if for every $s, t \in T$ of the same length,

$$
s \frown 0 \in T \leftrightarrow t \frown 0 \in T \text { and } s \frown 1 \in T \leftrightarrow t \frown 1 \in T .
$$

A branch of a tree $T \subseteq 2^{<\omega}$ is an infinite sequence $c \in 2^{\omega}$ such that for every $n, c\left\lceil n \in T\right.$. We denote by $[T]$ the set of branches of $T$. A tree $T \subseteq 2^{<\omega}$ 
is perfect if for every $t \in T$ there is $s \in T$ with $t \sqsubseteq s$ for which $s \frown 0 \in T$ and $s \frown 1 \in T$.

It is clear that every perfect uniform tree $T \subseteq 2^{<\omega}$ determines a sublattice $[A, B]$. Namely, $A=\{n \in \omega: \forall c \in[T](c(n)=1)\}$ and $B=\{n \in \omega$ : $\exists c \in[T](c(n)=1)\}$

We will show that $\omega \rightarrow((\omega))^{\omega}$ holds in $N_{1}$. For this we prove a more general result.

Theorem 9. Let $M$ be a transitive model of $Z F C$, let $M\left[\left\{a_{\xi}: \xi<\omega_{1}\right\}\right]$ be the generic extension obtained using the forcing notion $\mathbb{C}_{\omega_{1}}$ which adds $\omega_{1}$ Cohen reals, and let $N=L\left(\left\{a_{\xi}: \xi<\omega_{1}\right\}\right)$ computed in the generic extension $M\left[\left\{a_{\xi}: \xi<\omega_{1}\right\}\right]$. In $N$ the property $\omega \rightarrow((\omega))^{\omega}$ holds.

Proof. Notice that if $y \in M\left[\left\{a_{\xi}: \xi<\omega_{1}\right\}\right]$ is Cohen generic over $M$, then the forcing $\mathbb{C}_{\omega_{1}}$ can be factored as $\mathbb{C} \times \mathbb{C}_{\omega_{1}}$, where $\mathbb{C}$ is Cohen forcing. So,

$$
M\left[\left\{a_{\xi}: \xi<\omega_{1}\right\}\right]=M[y]\left[G^{\prime}\right],
$$

where $G^{\prime}$ is $\mathbb{C}_{\omega_{1}}$-generic over $M[y]$.

We will prove that if $\mathcal{A} \subseteq[\omega]^{\omega}$ in $N$, then there is a sublattice $[K, H] \in N$ such that $[K, H] \subseteq \mathcal{A}$ or $[K, H] \cap \mathcal{A}=\emptyset$.

Let $\mathcal{A} \subseteq[\omega]^{\omega}$ be such that $\mathcal{A} \in N$, and let $\alpha=\operatorname{rank}_{N}(\mathcal{A})$. There is a formula $\Phi\left(z, a_{\delta_{0}}, \ldots, a_{\delta_{k-1}}\right)$ that defines $\mathcal{A}$.

By the factoring mentioned at the beginning of the proof, we can assume that the parameters $a_{\delta_{i}}(i<k)$ of the formula are in the base model $M$.

Let $\mathbb{P}$ be the forcing of finite uniform subtrees of $2^{<\omega}$ ordered by end extension, thus, $T_{2} \leq T_{1}$ if and only if $T_{1} \subseteq T_{2}$ and $T_{2}\left\lceil\right.$ height $\left(T_{1}\right)=T_{1}$. Here height $\left(T_{1}\right)$ is the length of any branch of $T_{1}$ (all branches of a finite uniform tree have the same length).

Since $\mathbb{P}$ is countable, it is Cohen forcing, therefore there are $\mathbb{P}$-generic filters over $M$ in $M\left[\left\{a_{\xi}: \xi<\omega_{1}\right\}\right]$. A $\mathbb{P}$-generic over $M$ is a perfect uniform tree, thus it is a sublattice $[A, B]$.

Claim 10. Every element of $[A, B]$ is Cohen generic over $M$.

Proof of claim. Take $x \in[A, B]$, and consider the filter generated by $x$, $\{x\lceil F: F \subset \omega$ finite $\}$. Let $D$ be a dense open subset of the Cohen forcing (in $M$ ). Define $D^{\prime}$ to be the set of all finite uniform perfect trees with all branches in $D$.

The following standard construction shows that $D^{\prime}$ is dense open in $\mathbb{P}$. Given $p \in \mathbb{P}$, a finite uniform binary tree, list the branches of $p$ as $\left\{b_{0}, b_{1}, \ldots, b_{k}\right\}$. Extend $b_{0}$ to some $b_{0}^{1} \in D$ so that $b_{0}^{1}=b_{0} \frown c_{0}$. For $j \leq k$ put $b_{j}^{1}=b_{j} \frown c_{0}$.

Continue extending $b_{1}^{1}$ to $b_{1}^{2} \in D$ with $b_{1}^{2}=b_{1}^{1} \frown c_{1}=b_{1} \frown c_{0} \frown c_{1}$. For all $j \leq k$ put $b_{j}^{2}=b_{j}^{1} \frown c_{1}$. Notice that $b_{0}^{2} \in D$ since $D$ is open and $b^{1} \in D$. 
Suppose we have defined $b_{0}^{m}, b_{1}^{m}, \ldots, b_{k}^{m}$ for $m<k$ so that for each $j \leq k$, $b_{j}^{m}=b_{j} \frown c_{1} \frown \cdots \frown c_{m-1}$, and for $j \leq m, b_{j}^{m} \in D$.

Extend now $b_{m+1}^{m}$ to $b_{m+1}^{m+1} \in D$ such that $b_{m+1}^{m+1}=b_{m+1}^{m} \frown c_{m}$, and put $b_{j}^{m+1}=b_{j}^{m} \frown c_{m}$ for every $j \leq k$.

This completes the inductive definition of $b_{0}^{m}, \ldots, b_{k}^{m}$ for all $m \leq k$. Clearly, the tree $q$ whose branches are $b_{0}^{k}, \ldots, b_{k}^{k}$ end-extends $p$, is uniform and belongs to $D^{\prime}$. So, there is $T \in D^{\prime}$ such that $T$ is in the $\mathbb{P}$-generic filter determined by $[A, B]$, i.e. $T \sqsubset[A, B]$. Thus there is $k$ such that $x\lceil k$ is a branch of $T$, and so $x\lceil k \in D$. Claim

We will see that for some $\left[A^{\prime}, B^{\prime}\right] \subseteq[A, B]$, we have $\left[A^{\prime}, B^{\prime}\right] \subseteq \mathcal{A}$ or $\left[A^{\prime}, B^{\prime}\right] \cap \mathcal{A}=\emptyset$. Take $x \in[A, B]$. If $M\left[\left\{a_{\xi}: \xi<\omega_{1}\right\}\right] \vDash x \in \mathcal{A}$, then in $M[x]$, by the weak homogeneity of the partial order $\mathbb{C}_{\omega_{1}}$, we have

$$
\emptyset \Vdash_{\mathbb{C}_{\omega_{1}}} \check{x} \in \mathcal{A},
$$

i.e., the empty condition forces $\check{x} \in \mathcal{A}$, in the forcing $\mathbb{C}_{\omega_{1}}$, where $\check{x}$ is the canonical name for $x$ as element of the base model $M[x]$.

Therefore, in $M$, there is a Cohen condition $p$ such that

$$
p \Vdash " \emptyset \Vdash_{\mathbb{C}_{\omega_{1}}} \check{\Gamma} \in \mathcal{A} " .
$$

Here $\Gamma$ is the canonical name for the $\mathbb{C}$-generic, and we admit a slight abuse of notation by writing $\check{\Gamma}$ for the canonical name for the object named by $\Gamma$.

Consider $[A, B]\left\lceil p\right.$, i.e. the sublattice $\left[A^{\prime}, B^{\prime}\right]=\{y \in[A, B]: p \sqsubset y\}$. Notice that $A^{\prime}$ differs from $A$ just by a finite set, and the same for $B^{\prime}$ and $B$. For every $y \in\left[A^{\prime}, B^{\prime}\right]$, since $p \Vdash " \emptyset \Vdash_{\mathbb{C}_{\omega_{1}}} \check{\Gamma} \in \mathcal{A}$ ", we have

$$
M[y] \vDash " \emptyset \Vdash_{\mathbb{C}_{\omega_{1}}} \check{y} \in \mathcal{A} " .
$$

Since the forcing that extends $M[y]$ to the full extension $M\left[\left\{a_{\xi}: \xi<\omega_{1}\right\}\right]$ is (isomorphic to) $\mathbb{C}_{\omega_{1}}$, we have

$$
M\left[\left\{a_{\xi}: \xi<\omega_{1}\right\}\right]=M[y]\left[G^{\prime}\right] \vDash y \in \mathcal{A} .
$$

We proceed in the same fashion if $M\left[\left\{a_{\xi}: \xi<\omega_{1}\right\}\right] \vDash x \notin \mathcal{A}$.

It remains to show that there is such a homogeneous sublattice $[A, B]$ in the model $N$. We can code finite uniform subtrees of $2^{<\omega}$ by finite sequences of 0 's and 1's, i.e. by elements of $2^{<\omega}$, in a canonical way. Dense subsets of the partial order $\mathbb{P}$ will correspond to dense subsets of the Cohen partial order. This means that any Cohen generic in the extension codes a generic sublattice. Therefore, we will have generic sublattices in the model $N$.

Notice that from this proof it follows that in the model $N$, every $\mathcal{A} \subseteq$ $[\omega]^{\omega}$ is Silver measurable, since we can reproduce this argument below any arbitrary $[C, D]$.

This argument is similar to the one used in [3] for projective partitions. 
Corollary 11. In the model $N=L\left(\left\{a_{\xi}: \xi<\omega_{1}\right\}\right)$ of the previous theorem, every set $\mathcal{A} \subseteq[\omega]^{\omega}$ contains or is disjoint from a perfect subset of $[\omega]^{\omega}$, i.e., there are no Bernstein sets in this model.

DeFinition 12. Let $M$ be a transitive model of ZFC, and $M[G]$ a generic extension. A real $x \in \omega^{\omega}$ is eventually different if for every $y \in \omega^{\omega} \cap M$ there is $k \in \omega$ such that $x(i) \neq y(i)$ for every $i \geq k$.

Observation. Let $M$ be a transitive model of ZFC, and let $M[g]$ be a Cohen extension. Then the Cohen generic real $g$ is not eventually different. This is because given $y \in M$ and $k \in \omega$, the set $D_{k}=\{p: \exists i \geq k(p(i)=$ $y(i))\}$ is dense. Moreover, for every $y \in M$ and $k \in \omega$, the set $E_{k}=\{p$ : $\exists i \geq k(p(i)=y(i)$ and $p(i+1)=y(i+1))\}$ is also dense, and therefore, for every $y \in M, g(i)=y(i)$ and $g(i+1)=y(i+1)$ for infinitely many $i$.

Observation. In a Cohen extension $M[g]$ no eventually different reals are added. To see this, let $r \in \omega^{\omega}$ be such that $r \in M[g] \backslash M$, and let $\dot{r}$ be a $\mathbb{C}$-name for $r$ in $M$. Enumerate the conditions of Cohen forcing $\mathbb{C}$ by $p_{0}, p_{1}, p_{2}, \ldots$ Define $x \in \omega^{\omega}$ by

$$
x(n):=\min \left\{k: \exists q \leq p_{n}(q \Vdash \dot{r}(\check{n})=\check{k})\right\} .
$$

Then $x \in M$, and we will see that $\{i \in \omega: r(i)=x(i)\}$ is infinite. Suppose not; then $\exists n_{0} \in \omega\left[\forall n \geq n_{0}(r(n) \neq x(n))\right]$. By the Forcing Theorem there is $p$ such that $p \Vdash \forall n \geq \check{d}(\dot{r}(n) \neq \check{x}(n))$. Pick $m>d$ such that $p_{m} \leq p$. If $x(m)=k$, then (by definition) $\exists q \leq p_{m}[q \Vdash \dot{r}(\check{m})=\check{k}]$. Therefore $q \Vdash \dot{r}(\check{m})=\check{x}(\check{m})$, which is a contradiction.

Once we know that $\forall x \in M[g] \exists y \in M(\{i \in \omega: x(i)=y(i)\}$ is infinite), it is easy to see that for all $x \in M[g]$ there is $y \in M$ such that $\{i \in \omega: x(i)=y(i) \wedge x(i+1)=y(i+1)\}$ is infinite: Let $r_{1} \in \omega^{\omega}$ be such that $r_{1} \in M[g] \backslash M$. Then consider the real $r_{2}$ which codifies the consecutive pairs of $r_{1}$. Apply to $r_{2}$ the previous result to find in $M$ a real $r_{3}$ such that $\left\{i \in \omega: r_{2}(i)=r_{3}(i)\right\}$ is infinite. Decoding from $r_{3}$ we obtain a real $r_{3}^{*}$ in $M$ such that the set $\left\{i \in \omega: r_{1}(i)=r_{3}^{*}(i) \wedge r_{1}(i+1)=r_{3}^{*}(i+1)\right\}$ is infinite.

Finally observe that if $M[G]$ is obtained by adding $\omega_{1}$ many Cohen reals, then no eventually different reals are added, because every new real is added by countably many Cohen generic reals $([12])$, thus by one Cohen generic real.

THEOREM 13 (Brendle). The polarized partition relation

$$
\left(\begin{array}{c}
\omega \\
\omega \\
\vdots
\end{array}\right) \rightarrow\left(\begin{array}{c}
2 \\
2 \\
\vdots
\end{array}\right)
$$

fails in $N_{1}$. 
Recall that $N_{1}$ is the model obtained adding $\omega_{1}$ many Cohen reals to $L$, and then computing $L\left(\left\{a_{\xi}: \xi<\omega_{1}\right\}\right)$ in the generic extension.

Proof. Since $L[G]$ does not contain eventually different reals,

$$
\forall x \in L[G] \exists y \in L(\{i \in \omega: x(i)=y(i)\} \text { is infinite }) .
$$

Moreover, for every $x \in L[G]$, there is $y \in L$ such that the set

$$
\{i \in \omega: x(i)=y(i) \text { and } x(i+1)=y(i+1)\}
$$

is infinite, as observed in the paragraphs preceding the statement of the theorem.

For every $x$, there is a first $y \in L$, in the $\boldsymbol{\Sigma}_{2}^{1}$ ordering of the reals in $L$, for which $\{i \in \omega: x(i)=y(i)$ and $x(i+1)=y(i+1)\}$ is infinite. We call it $f(x)$. Let $i_{x}$ be the first $i$ such that $x(i)=f(x)(i)$.

Define $c: \omega^{\omega} \rightarrow 2$ by $c(x)=0$ if and only if $i_{x}$ is even.

We show that no product of pairs is monochromatic for $c$. In $L[G]$, let $\left\{H_{i}\right\}_{i \in \omega}$ be a sequence of pairs of integers, and let $x \in \prod_{i \in \omega} H_{i}$. Notice that we can assume that $x\left(i_{x}\right)=f(x)\left(i_{x}\right)$ and $x\left(i_{x}+1\right)=f(x)\left(i_{x}+1\right)$, since we can modify a finite number of values of $x$ and obtain another member of the product with the same $f(x)$ satisfying this requirement.

Now, modifying the $i_{x}$ th value of $x$ we obtain $x^{\prime} \in \prod_{i \in \omega} H_{i}$ such that $c(x) \neq c\left(x^{\prime}\right)$.

The coloring $c$ is $\boldsymbol{\Delta}_{2}^{1}$, since $c^{-1}\{0\}$ and $c^{-1}\{1\}$ are both $\boldsymbol{\Pi}_{2}^{1}$. To see this, note that $c(x)=0$ if and only if the first $i$ such that $x(i)=f(x)(i)$ is even; and the function $f$ is defined by a $\Pi_{2}^{1}$ formula: given $x, z=f(x)$ if and only if $\forall y\left(y \leq_{L} z \Rightarrow\{i: z(i)=x(i)\right.$ and $z(i+1)=x(i+1)\}$ is finite $)$.

We see that $M[G]$ has the property that for every sequence $\left\{H_{i}\right\}_{i \in \omega}$ of pairs of natural numbers, there are $x, y \in \prod_{i \in \omega} H_{i}$ such that $c(x) \neq c(y)$.

Since $c$ is definable, it is in $N_{1}=L\left(\left\{a_{\xi}: \xi<\omega_{1}\right\}\right)$, and in this model the above property holds. Thus the polarized partition property does not hold in $N_{1}$.

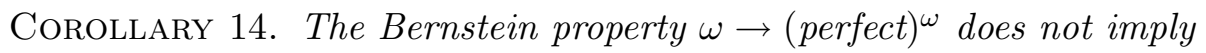

$$
\left(\begin{array}{c}
\omega \\
\omega \\
\vdots
\end{array}\right) \rightarrow\left(\begin{array}{c}
2 \\
2 \\
\vdots
\end{array}\right) .
$$

Neither of the properties $\omega \rightarrow((\omega))^{\omega}$ and

$$
\left(\begin{array}{c}
\omega \\
\omega \\
\vdots
\end{array}\right) \rightarrow\left(\begin{array}{c}
2 \\
2 \\
\vdots
\end{array}\right)
$$

implies the other. 
Proof. The model $N_{1}$ considered above satisfies $\omega \rightarrow((\omega))^{\omega}$ (and there-

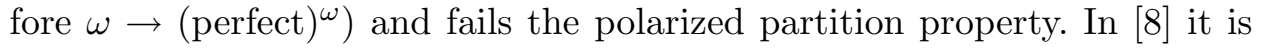
shown that a selective ultrafilter can be added generically to a Solovay model $L(\mathbb{R})$ to obtain a model $L(\mathbb{R})[U]$ in which the polarized partition property holds but $\omega \rightarrow((\omega))^{\omega}$ does not.

Clearly,

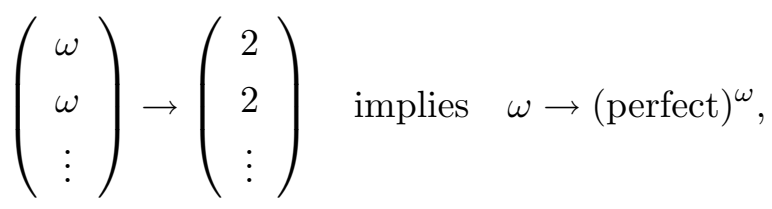

since a product of the form $\prod_{i \in \omega} H_{i}$, with $\left\{i \in \omega:\left|H_{i}\right| \geq 2\right\}$ infinite, is a perfect set. But the previous results show that the reverse implication does not hold, considering that

$$
\omega \rightarrow((\omega))^{\omega} \text { implies } \omega \rightarrow{\text { (perfect })^{\omega},}^{\omega}
$$

since any $[K, H]$ is a perfect subset of $[\omega]^{\omega}$. This answers a question in [5].

4. A model with an $\aleph_{1}$-sequence of reals. We will now describe a model of the form $L(A)$ for a certain set $A$, where there is a non-meager filter on $\omega$, there is an $\aleph_{1}$-sequence of real numbers, and the sublattice property holds.

Let $L\left[\left\{a_{\beta}: \beta<\omega_{2}\right\}\right]$ be a generic extension of $L$ obtained by forcing with the Cohen order $\mathbb{C}_{\aleph_{2}}$ adding $\aleph_{2}$ generic reals. Let $A=\left\{\left\langle a_{\xi}: \xi \in \alpha\right\rangle\right.$ : $\left.\alpha<\aleph_{2}\right\} \cup\left\{a_{\xi}: \xi<\aleph_{2}\right\}$, and consider the model $N_{2}=L(A)$ built in $L\left[\left\{a_{\beta}\right.\right.$ : $\left.\beta<\omega_{2}\right\}$ ]. It can be shown, just as in Section 2, that $A \notin N_{2}$ and $N_{2}$ does not satisfy the Axiom of Choice. Clearly, in $N_{2}$ there is an $\aleph_{1}$-sequence of reals.

THEOREM 15. In $N_{2}$ there is a non-meager filter on $\omega$.

Proof. Consider the filter $\mathcal{F}$ on $\omega$ generated in $N_{2}$ by $\left\{a_{\xi}: \xi<\aleph_{1}\right\}$ and the cofinite sets. We show that $\mathcal{F}$ is non-meager. Let $\left\{T_{i}: i \in \omega\right\}$ be a sequence of nowhere dense closed sets, and suppose, aiming for a contradiction, that $\bigcup_{i \in \omega} T_{i}$ covers $\mathcal{F}$.

Each $T_{i}$ is in a model of the form $L\left[G\lceil B]\right.$ where $B \subseteq \aleph_{2}$ is countable, $G\left\lceil B:=\left\{p \in \mathbb{C}_{B}: p \in G\right\}\right.$, where $\mathbb{C}_{B}:=\left\{p \in \mathbb{C}_{\aleph_{2}}: \operatorname{dom}(p) \subseteq B \times \omega\right\}$. This is because each $T_{i}$ can be coded by a real. Moreover, there is a countable set $D$ of generic reals such that $\left\{T_{i}: i \in \omega\right\}$, and therefore $\mathcal{F}$, belongs to $L[G \nmid D]$. But then there is a generic real $a_{\xi}\left(\right.$ in $\left.N_{2}\right), \xi<\aleph_{1}$, which is generic over $L\left[G\lceil D]\right.$. This is a contradiction since $a_{\xi}$ is an element of $\mathcal{F}$.

Theorem 16. The model $N_{2}$ has the sublattice property $\omega \rightarrow((\omega))^{\omega}$.

Proof. That the property $\omega \rightarrow((\omega))^{\omega}$ holds in $N_{2}$ is shown just as for $N_{1}$ (see proof of Theorem 9). 
This shows that although $\omega \rightarrow((\omega))^{\omega}$ contradicts the existence of a nonprincipal ultrafilter on $\omega$, it is consistent with the existence of a non-meager filter on that set.

If $\aleph_{1} \leq 2^{\omega}$, there is an uncountable set of real numbers without perfect subsets. This contrasts with the following corollary.

COROllary 17. $\aleph_{1} \leq 2^{\omega}$ does not imply the existence of a Bernstein set.

Proof. By Theorem 16 and the fact that $\omega \rightarrow((\omega))^{\omega}$ implies $\omega \rightarrow$ (perfect) ${ }^{\omega}$.

It is known that the existence of a non-principal ultrafilter on $\omega$ provides a counterexample to $\omega \rightarrow(\omega)^{\omega}$ [13]. In [4] a model is given where there is a non-principal ultrafilter on $\omega$ and the Bernstein property holds. A stronger result was obtained in [8], with a model for a non-principal ultrafilter on $\omega$ and the polarized property. We end this section by observing that the existence of a non-meager filter on $\omega$ contradicts $\omega \rightarrow(\omega)^{\omega}$ (see [13]).

Lemma 18 (Jalali-Naini and Talagrand, see [16]). The following are equivalent for every non-principal filter $\mathcal{F}$ on $\omega$ :

(1) $\mathcal{F}$ is non-meager.

(2) For every infinite sequence $n_{0}<n_{1}<\cdots$ of natural numbers there is an element $C$ of $\mathcal{F}$ such that $\left\{i \in \omega: C \cap\left[n_{i}, n_{i+1}\right)=\emptyset\right\}$ is infinite.

THEOREM 19. If there is a non-meager filter on $\omega$ then $\omega \nrightarrow(\omega)^{\omega}$.

Proof. Let $\mathcal{F}$ be a non-meager filter on $\omega$ containing the cofinite sets. Define $c:[\omega]^{\omega} \rightarrow 2$ as follows. If $A=\left\{n_{0}, n_{1}, \ldots\right\}$ is listed increasingly, then $c(A)=0$ if and only if $\bigcup_{i \in \omega}\left[n_{2 i}, n_{2 i+1}\right)$ belongs to $\mathcal{F}$.

No set of the form $[A]^{\omega}$ is homogeneous for $c$. To see this, let $A=$ $\left\{n_{0}, n_{1}, \ldots\right\}$ be listed increasingly. By Lemma 18 there is a set $C \in \mathcal{F}$ and an infinite set $I \subseteq \omega$ such that $C$ is disjoint from the interval $\left[n_{i}, n_{i+1}\right)$ if and only if $i \in I$. Let $B \subseteq A$ be obtained inductively as follows. Let $n_{i_{0}}=n_{0}$ and, if $n_{i_{j}}$ has been defined, let $i_{j+1}$ be the least $m>i_{j}$ such that $n_{m} \in I$ if and only if $n_{i_{j}} \notin I$. Put $B=\left\{n_{i_{j}}: j \in \omega\right\}$.

Now, $c$ takes different values in $B$ and $B \backslash\{\min (B)\}$, because $C$ is contained in the union of the even intervals determined by $B$ if and only if it is almost contained in the union of the odd intervals determined by $B \backslash\{\min (B)\}$, and vice versa, $C$ is contained in the union of the odd intervals determined by $B$ if and only if it is almost contained in the union of the even intervals determined by $B \backslash\{\min (B)\}$.

Acknowledgments. We thank Joerg Brendle and Stevo Todorcevic for several conversations and suggestions. 


\section{References}

[1] F. Bernstein, Zur Theorie der Trigonometrischen Reihen, Ber. Verhandlungen Königlich Sächsischen Gesellschaft Wiss. Leipzig Math.-Phys. Kl. 60 (1908), 325-338.

[2] A. Blass, The model of set theory generated by countably many generic reals, J. Symbolic Logic 46 (1981), 732-752.

[3] J. Brendle, L. Halbeisen and B. Löwe, Silver measurability and its relation to other regularity properties, Math. Proc. Cambridge Philos. Soc. 138 (2005), 135-149.

[4] C. A. Di Prisco, Partition properties and perfect sets, Notas Lógica Mat 38 (1993), 119-127.

[5] - Mathematics versus metamathematics in Ramsey theory of the real numbers, in: Logic, Methodology and Philosophy of Science (Oviedo, 2003), P. Hajek et al. (eds.), Kings College Publ., London, 2005, 171-187.

[6] C. A. Di Prisco and J. Henle, Doughnuts, floating ordinals, square brackets and utrafilters, J. Symbolic Logic 65 (2000), 461-473.

[7] - - - Partitions of reals and choice, in: Models, Algebras and Proofs, X. Caicedo and C. Montenegro (eds.), Lecture Notes in Pure Appl. Math. 203, Dekker, 1999, $13-23$.

[8] C. A. Di Prisco and S. Todorcevic, Suslin partition of products of finite sets, Adv. Math. 176 (2003), 172-183.

[9] S. Feferman, Some applications of the notions of forcing and generic sets, Fund. Math. 56 (1965), 325-345.

[10] L. Halbeisen, Making doughnuts of Cohen reals, Math. Logic Quart. 49 (2003), 173-178.

[11] K. Kunen, Set Theory. An Introduction to Independence Proofs, North-Holland, 1980 .

[12] A. Levy, Definability in axiomatic set theory II, in: Mathematical Logic and Foundations of Set Theory (Jerusalem, 1968), Y. Bar-Hillel (ed.), North-Holland, 1970, $129-145$.

[13] A. R. D. Mathias, A remark on rare filters, in: A. Hajnal et al. (eds.), Infinite and Finite Sets, Colloq. Math. Soc. Janos Bolyai 10, North-Holland, Amsterdam, 1975, 1095-1097.

[14] —, Happy families, Ann. of Math. Logic 12 (1977), 59-111.

[15] S. Shelah, can you take Solovay's inaccessible away? Israel J. Math. 48 (1984), 1-47.

[16] S. Todorcevic, Topics in Topology, Lecture Notes in Math. 1652, Springer, 1997.

[17] J. Truss, Models of set theory containing many perfect sets, Ann. of Math. Logic 7 (1974), 197-219.

Carlos Augusto Di Prisco

Departamento de Matemáticas

Instituto Venezolano

de Investigaciones Científicas

Apartado 20632

Caracas 1020-A, Venezuela

E-mail: cdiprisc@ivic.gob.ve
Franklin C. Galindo Departamento de Lógica y Filosofía de la Ciencia

Escuela de Filosofía Facultad de Humanidades y Educación Universidad Central de Venezuela Apartado 47209.A Caracas 1041-A, Venezuela E-mail: franklin.galindo@ucv.ve

Received 1 July 2009; in revised form 4 February 2010 